\title{
Light meets water in nonlocal media: Surface tension analogue in optics
}

\author{
Theodoros P. Horikis ${ }^{1}$ and Dimitrios J. Frantzeskakis ${ }^{2}$ \\ ${ }^{1}$ Department of Mathematics, University of Ioannina, Ioannina 45110, Greece \\ ${ }^{2}$ Department of Physics, National and Kapodistrian University of Athens, Panepistimiopolis, Zografos, Athens 15784, Greece
}

\begin{abstract}
Shallow water wave phenomena find their analogue in optics through a nonlocal nonlinear Schrödinger (NLS) model in $(2+1)$-dimensions. We identify an analogue of surface tension in optics, namely a single parameter depending on the degree of nonlocality, which changes the sign of dispersion, much like surface tension does in the shallow water wave problem. Using multiscale expansions, we reduce the NLS model to a KadomtsevPetviashvilli (KP) equation, which is of the KPII (KPI) type, for strong (weak) nonlocality. We demonstrate the emergence of robust optical antidark solitons forming Y-, X- and H-shaped wave patterns, which are approximated by colliding KPII line solitons, similar to those observed in shallow waters.
\end{abstract}

PACS numbers: 42.65.-k, 05.45.Yv, 42.65.Tg, 47.11.St, 47.35.Fg

Many physically different contexts can be brought together through common modeling and mathematical description. A common (and rather unlike) example is water waves and nonlinear optics. Two models are inextricably linked with both subjects: the universal Korteweg-de Vries (KdV) and nonlinear Schrödinger (NLS) equations [1]. Furthermore, these models can be reduced from one to the other [2], thus suggesting that phenomena occurring in water waves may also exist in optics. Here, using such reductions for a nonlocal NLS, we find that surface tension - which causes fluids to minimize the area they occupy- has a direct analogue in optics.

Key to our findings are solitons, i.e., robust localized waves that play a key role in numerous studies in physics [3], applied mathematics [4] and engineering [5]. A unique property of solitons is that they feature a particle-like character, i.e., they interact elastically, preserving their shapes and velocities after colliding with each other. Such elastic collisions, as well as pertinent emerging wave patterns, can sometimes be observed even in everyday life. A predominant example is the one pertaining to flat beaches: in such shallow water wave settings, two line solitons merging at proper angles give rise to patterns of X-, H-, or Y-shaped waves, as well as other more complicated nonlinear waveforms [6]. All these shallow water wave structures are actually exact analytical multi-dimensional line soliton solutions of the Kadomtsev-Petviashvilli (KP) equation (which generalizes KdV to two dimensions (2D) [1, 4]) of the KPII type - a key model in the theory of shallow water waves with weak surface tension [1]. The relative equation with strong surface tension is referred to as KPI.

Here we show that such patterns can also be observed in a quite different physical setting, i.e., the one related to optical beam propagation in media with a spatially nonlocal defocusing nonlinearity. Such media include thermal nonlinear optical media [7, 8], partially ionized plasmas [9, 10], nematic liquid crystals [11, 12], and dipolar bosonic quantum gases [13]. It is shown that approximate solutions of the nonlocal NLS model satisfy, at proper scales, equations that appear in the context of water waves: a Boussinesq or Benney-Luke (BL) [14], as well as a KP equation. For a relatively strong (weak) nonlocality, or background amplitude, the latter is found to be a KPII (KPI), similarly to the water wave problem, where a
KPII (KPI) results in the case of small (large) surface tension [1]. Our results thus suggest an analogue of surface tension in optics. Direct numerical simulations show that approximate antidark line soliton solutions of the nonlocal NLS, constructed from the KPII line soliton solutions, form patterns observable in shallow water [6]. Pertinent Y-, X-, or H-wave patterns may be realized in an experimental setup similar to the one used for the observation of antidark solitons [15].

The evolution of optical beams in nonlinear defocusing media is governed by the following paraxial wave equation (cf. Ref. [16] for derivation and relevant adimensionalizations):

$$
i u_{t}+\frac{1}{2} \Delta u-n u=0,
$$

where subscripts denote partial derivatives, $u$ is the complex electric field envelope, $\Delta \equiv \partial_{x}^{2}+\partial_{y}^{2}$ is the transverse Laplacian, and real function $n$ denotes the nonlinear, generally nonlocal, medium response. For instance, in optics, $n$ is the nonlinear change of the refractive index depending on the intensity $I=|u|^{2}[7,8]$, in plasmas is the relative electron temperature perturbation [9, 10], in liquid crystals is the optically induced angle perturbation [11, 12], and so on. Here, we consider that $n$ obeys the following diffusion-type equation:

$$
d^{2} \Delta n-n+|u|^{2}=0,
$$

where $d$ is a spatial scale (setting the diffusion length) that measures the degree of nonlocality. Note that for $d=0$, Eqs. (1)-(2) reduce to the defocusing 2D NLS equation [16]. Importantly, Eqs. (1)-(2), the nonlocal NLS model of principal interest herein, has been used satisfactorily to model experiments on liquid solutions exhibiting thermal nonlinearities [17, 18], while it has also been used in studies of plasmas [9, 10] and nematic liquid crystals [11, 12].

The steady-state solution of Eqs. (1)-(2) is composed by the continuous wave (cw), $u=u_{0} \exp \left(-i\left|u_{0}\right|^{2} t\right)\left(u_{0}\right.$ being an arbitrary complex constant), and the constant function $n=\left|u_{0}\right|^{2}$. Considering small perturbations of this solution behaving like $\exp [i(\boldsymbol{k} \cdot \boldsymbol{r}-\omega t)]$, with $\boldsymbol{r}=(x, y)$, we find that the perturbations' wavevector $\boldsymbol{k}=\left(k_{x}, k_{y}\right)$ and frequency $\omega$ obey the dispersion relation:

$$
\omega^{2}=|\boldsymbol{k}|^{2} C^{2}\left(1+d^{2}|\boldsymbol{k}|^{2}\right)^{-1}+(1 / 4)|\boldsymbol{k}|^{4},
$$


where $C^{2}=\left|u_{0}\right|^{2}$ is the wave velocity. Here it is important to observe the following. First, since $\omega \in \mathbb{R} \forall \boldsymbol{k}$, the steadystate solution is modulationally stable. Second, in the longwavelength limit $\left(|\boldsymbol{k}|^{2} \ll 1\right)$, Eq. 3 becomes $\omega^{2} \approx|\boldsymbol{k}|^{2} C^{2}+$ $(1 / 4) \alpha|\boldsymbol{k}|^{4}$, where $\alpha=1-4 \bar{d}^{2}\left|u_{0}\right|^{2}$. This approximate dispersion relation features a striking similarity to the corresponding (approximate) one for shallow water waves, namely [1]: $\omega^{2} \approx|\boldsymbol{k}|^{2} c_{0}^{2}+(1 / 3)(3 \hat{T}-1) c_{0}^{2} h^{2}|\boldsymbol{k}|^{4}$, where $c_{0}^{2}=g h$ is the velocity, $g$ is the acceleration of gravity, $h$ the depth of water at rest, and $\hat{T}=T /\left(\rho g h^{2}\right)$, with $\rho$ being the density and $T$ the surface tension. Comparing these dispersion relations, the following correspondence is identified: $3 \hat{T} \rightarrow 4 d^{2}\left|u_{0}\right|^{2}$, implying that there exists a surface tension analogue in our problem, $\propto d^{2}\left|u_{0}\right|^{2}$. This effective surface tension is negative, as is also implied by the fact that the term $\propto d$ in the Hamiltonian $\mathcal{H}=(1 / 2) \int_{\mathbb{R}^{2}}\left(|\boldsymbol{\nabla} u|^{2}-d^{2}(\boldsymbol{\nabla} n)^{2}-n^{2}+2 n|u|^{2}\right) \mathrm{d} \boldsymbol{r}$ of Eqs. (1)-(2) decreases the potential energy of the system, oppositely to the water wave case where surface tension increases the respective potential energy [19].

These arguments can further be solidified by analyzing the fully nonlinear problem: similarly to water waves [1], we will derive KPI and KPII equations, depending on the strength of the effective surface tension, i.e., the parameter $\alpha$, which sets the dispersion coefficient in KP. This can already be identified from the linear theory as follows. Using $|\boldsymbol{k}|^{2}=k_{x}^{2}+k_{y}^{2}$, the long-wavelength limit of Eq. (3) reads: $\omega= \pm C k_{x}\left[1+\left(k_{y} / k_{x}\right)^{2}\right]^{1 / 2}\left[1+\left(\alpha / 4 C^{2}\right) k_{x}^{2}+\mathcal{O}\left(k_{y}^{2}\right)\right]$, with \pm corresponding to right- and left-going waves. Assuming $\left|k_{y} / k_{x}\right| \ll 1$ and $k_{y}^{2} \sim \mathcal{O}\left(k_{x}^{4}\right)$, we find: $(1 / C) \omega k_{x}=$ $\pm\left[k_{x}^{2}+\left(\alpha / 4 C^{2}\right) k_{x}^{4}+(1 / 2) k_{y}^{2}\right]$. Then, using $\omega \rightarrow i \partial_{t}$, $k_{x, y} \rightarrow-i \partial_{x, y}$, the linear PDE associated to this dispersion relation is: $\partial_{x}\left[ \pm q_{t}+C q_{x}-(\alpha / 8 C) q_{x x x}\right]+(C / 2) q_{y y}=0$. This is a linear KP equation, with a dispersion coefficient depending on the effective surface tension through $\alpha$, similarly to shallow water waves, where the respective dispersion coefficient depends on $\hat{T}[1]$.

To derive the full nonlinear version of the KP equation, we resort to multiple scales. We thus consider small-amplitude slowly-varying modulations of the steady state, and seek solutions of Eqs. (12)-(2) in the form of the asymptotic expansions:

$$
\begin{aligned}
& u=u_{0} \sqrt{\rho} \exp \left(-i\left|u_{0}\right|^{2} t+i \epsilon^{1 / 2} \Phi\right), \\
& \rho=1+\sum_{j=1}^{\infty} \epsilon^{j} \rho_{j}, \quad n=\left|u_{0}\right|^{2}+\sum_{j=1}^{\infty} \epsilon^{j} n_{j},
\end{aligned}
$$

where $0<\epsilon \ll 1$ is a formal small parameter, while phase $\Phi$ and amplitudes $\rho_{j}$ and $n_{j}$ are unknown real functions of the slow variables $X=\epsilon^{1 / 2} x, Y=\epsilon^{1 / 2} y$ and $T=\epsilon^{1 / 2} t$. Substituting the expansions (4)-(5) into Eqs. (1)-(2), we obtain the following results. First, the real part of Eq. (1) and Eq. (2) yield the leading-order equations, at $\mathcal{O}\left(\epsilon^{3 / 2}\right)$ and $\mathcal{O}(\epsilon)$ :

$$
\rho_{1 T}+\tilde{\Delta} \Phi=0, \quad n_{1}=\left|u_{0}\right|^{2} \rho_{1},
$$

and the first-order equations, at $\mathcal{O}\left(\epsilon^{5 / 2}\right)$ and $\mathcal{O}\left(\epsilon^{2}\right)$ :

$$
\rho_{2 T}+\tilde{\nabla} \cdot\left(\rho_{1} \tilde{\nabla} \Phi\right)=0, \quad d^{2} \tilde{\Delta} n_{1}-n_{2}+\left|u_{0}\right|^{2} \rho_{2}=0,
$$

connecting the amplitudes $\rho_{1,2}$ and $n_{1,2}$ with the phase $\Phi$; here, $\tilde{\Delta} \equiv \partial_{X}^{2}+\partial_{Y}^{2}$ and $\tilde{\nabla} \equiv\left(\partial_{X}, \partial_{Y}\right)$. Second, the imaginary part of Eq. (1), combined with Eqs. (6)-(7), yields:

$$
\begin{aligned}
& \Phi_{T T}-C^{2} \tilde{\Delta} \Phi+\epsilon\left[\frac{1}{4} \alpha \tilde{\Delta}^{2} \Phi+\frac{1}{2} \partial_{T}(\tilde{\nabla} \Phi)^{2}\right. \\
& \left.+\tilde{\nabla} \cdot\left(\Phi_{T} \tilde{\nabla} \Phi\right)\right]=\mathcal{O}\left(\epsilon^{2}\right),
\end{aligned}
$$

Equation (8) incorporates 4th-order dispersion and quadratic nonlinear terms, resembling the Boussinesq and Benney-Luke [14] equations, which describe bidirectional shallow water waves [1]. Similarly to the water wave problem, we now use a multiscale expansion method to derive the KP equation, under the additional assumptions of quasi-two-dimensionality and unidirectional propagation. In particular, we introduce the asymptotic expansion $\Phi=\Phi_{0}+\epsilon \Phi_{1}+\cdots$, where functions $\Phi_{\ell}(\ell=0,1, \ldots)$ depend on the variables $\xi=X-C T$, $\eta=X+C T, \mathcal{Y}=\epsilon^{1 / 2} Y$, and $\mathcal{T}=\epsilon T$. Substituting this expansion into Eq. (8), at the leading-order in $\epsilon$, we obtain the wave equation $\Phi_{0 \xi \eta}=0$, implying that $\Phi_{0}$ can be expressed as a superposition of a right-going wave, $\Phi_{0}^{(R)}$, depending on $\xi$, and a left-going one, $\Phi_{0}^{(L)}$, depending on $\eta$, namely:

$$
\Phi_{0}=\Phi_{0}^{(R)}(\xi, \mathcal{Y}, \mathcal{T})+\Phi_{0}^{(L)}(\eta, \mathcal{Y}, \mathcal{T}) .
$$

In addition, at order $\mathcal{O}(\epsilon)$, we obtain the equation:

$$
\begin{aligned}
& 4 C^{2} \Phi_{1 \xi \eta}=-C\left(\Phi_{0 \xi \xi}^{(R)} \Phi_{0 \eta}^{(L)}-\Phi_{0 \xi}^{(R)} \Phi_{0 \eta \eta}^{(L)}\right) \\
& +\left[\partial_{\xi}\left(-2 C \Phi_{0 \mathcal{T}}^{(R)}+\frac{\alpha}{4} \Phi_{0 \xi \xi \xi}^{(R)}-\frac{3 C}{2} \Phi_{0 \xi}^{(R) 2}\right)-C^{2} \Phi_{0 \mathcal{Y} \mathcal{Y}}^{(R)}\right] \\
& +\left[\partial_{\eta}\left(2 C \Phi_{0 \mathcal{T}}^{(L)}+\frac{\alpha}{4} \Phi_{0 \tilde{\eta} \tilde{\eta} \tilde{\eta}}^{(L)}+\frac{3 C}{2} \Phi_{0 \tilde{\eta}}^{(L) 2}\right)-C^{2} \Phi_{0 \mathcal{Y} \mathcal{Y}}^{(L)}\right] .
\end{aligned}
$$

When integrating this equation, secular terms arise from the square brackets, which are functions of $\xi$ or $\eta$ alone, not both. Removal of these secular terms leads to two uncoupled nonlinear evolution equations for $\Phi_{0}^{(R)}$ and $\Phi_{0}^{(L)}$. Then, using $\Phi_{T}=-n_{1}$, obtained from the leading-order part of Eq. (8) together with Eq. (7), it is found that the amplitude $\rho_{1}$ can also be decomposed to a left- and a right-going wave, i.e., $\rho_{1}=\rho_{1}^{(R)}+\rho_{1}^{(L)}$, which satisfy the following KP equations:

$\partial \mathcal{X}\left( \pm \rho_{1 \mathcal{T}}^{(R, L)}-\frac{\alpha}{8 C} \rho_{1 \mathcal{X} \mathcal{X} \mathcal{X}}^{(R, L)}+\frac{3 C}{4} \rho_{1}^{(R, L) 2}\right)+\frac{C}{2} \rho_{1 \mathcal{Y} \mathcal{Y}}^{(R, L)}=0$,

where $\mathcal{X}=\xi(\mathcal{X}=\eta)$ for the right- (left-) going wave. Next, for right-going waves, we use the transformations $\mathcal{T} \rightarrow$ $-(\alpha / 8 C) \mathcal{T}, \mathcal{Y} \rightarrow \sqrt{3|\alpha| / 4 C^{2}} \mathcal{Y}$, and $\rho_{1}^{(R)}=-\left(\alpha / 2 C^{2}\right) U$, and express $\mathrm{KP}$ in its standard dimensionless form [1, 4]:

$$
\partial_{\mathcal{X}}\left(U_{\mathcal{T}}+6 U U_{\mathcal{X}}+U_{\mathcal{X X X}}\right)+3 \sigma^{2} U_{\mathcal{Y Y}}=0,
$$

where $\sigma^{2}=-\operatorname{sgn} \alpha=\operatorname{sgn}\left(4 d^{2}\left|u_{0}\right|^{2}-1\right)$. Importantly, Eq. (10) includes both versions of the KP equation, KPI and KPII [4]. Indeed, for $\sigma^{2}=1 \Rightarrow \alpha<0$, i.e., for 
$4 d^{2}\left|u_{0}\right|^{2}>1$, Eq. 10 is a KPII equation; on the other hand, for $\sigma^{2}=-1 \Rightarrow \alpha>0$, i.e., $4 d^{2}\left|u_{0}\right|^{2}<1$, Eq. 10 is a KPI equation. Thus, for a fixed cw intensity $\left|u_{0}\right|^{2}$, a strong (weak) nonlocality $d^{2}$, as defined by the above inequalities, corresponds to KPII (KPI); the same holds for a fixed degree of nonlocality $d^{2}$, and a larger (smaller) cw intensity $\left|u_{0}\right|^{2}$. Thus, both our linear and nonlinear analysis establishes a "homeomorphism" between optics and shallow water waves: in this latter context, weak surface tension (typical for water waves) corresponds to $\sigma^{2}=1$ in Eq. 10 (i.e., to KPII), while strong surface tension is pertinent to $\sigma^{2}=-1$ (i.e., to KPI) [1, 4].

Based on the above analysis, we now utilize the exact solutions of the KP, Eq. (10), and construct approximate solutions of the original system of Eqs. (1)-(2); such solutions read:

$$
\begin{aligned}
& u \approx u_{0}\left(1-\epsilon \frac{\alpha}{2\left|u_{0}\right|^{2}} U\right)^{1 / 2} \exp \left(-i\left|u_{0}\right|^{2} t\right) \\
& \times \exp \left(\frac{i}{2} \alpha \epsilon^{-1 / 2} \int_{0}^{\mathcal{T}} U \mathrm{~d}^{\prime}\right), \quad n \approx\left|u_{0}\right|^{2}-\frac{1}{2} \alpha U .
\end{aligned}
$$

Clearly, for $\alpha<0(\alpha>0)$, i.e., for solutions satisfying KPII (KPI), $u$ in Eq. (11) has the form of a hump (dip) on top (off) of the cw background and is, thus, a antidark (dark) soliton.

Notice that in the local limit of $d=0$ (i.e., $\alpha=1$ ), we solely obtain the KPI model, in which line solitons are unstable: as was shown in plasma physics and hydrodynamics [20], line solitons develop undulations and eventually decay into lumps [21]. In the same venue, but now in optics, the asymptotic reduction of the defocusing 2D NLS to KPI [22, 23], and the instability of the line solitons of the latter, was used to better understand the transverse instability of rectilinear dark solitons: indeed, these structures also develop undulations and eventually decay into vortex pairs [23, 24].

Here, we focus on the stable soliton solutions of the KP equations, namely the (antidark) line solitons of the KPII equation and the (dark) lump of KPI. The one-line soliton solution, travelling at an angle to the $\mathcal{Y}$-axis, is:

$$
U(\mathcal{X}, \mathcal{Y}, \mathcal{T})=2 \kappa^{2} \operatorname{sech}^{2}(\mathcal{Z})
$$

where $\mathcal{Z} \equiv \kappa\left[\mathcal{X}+\lambda \mathcal{Y}-\left(4 \kappa^{2}+3 \lambda^{2}\right) \mathcal{T}+\delta\right]$, with $\kappa, \lambda$ and $\delta$ being free parameters. On the other hand, the two-line soliton can be expressed in the following form:

$$
\begin{gathered}
U=2 \partial_{\mathcal{X}}^{2} \ln \left(1+e^{\mathcal{Z}_{1}}+e^{\mathcal{Z}_{2}}+e^{\mathcal{Z}_{1}+\mathcal{Z}_{2}+A_{12}}\right), \\
\exp \left(A_{12}\right)=\frac{4\left(\kappa_{1}-\kappa_{2}\right)^{2}-\left(\lambda_{1}-\lambda_{2}\right)^{2}}{4\left(\kappa_{1}+\kappa_{2}\right)^{2}-\left(\lambda_{1}-\lambda_{2}\right)^{2}}
\end{gathered}
$$

where $\mathcal{Z}_{i} \equiv \kappa_{i}\left[\mathcal{X}+\lambda_{i} \mathcal{Y}-\left(4 \kappa_{i}^{2}+3 \lambda_{i}^{2}\right) \mathcal{T}+\delta_{i}\right]$

As was shown and observed in the context of shallow water waves [6], when two line solitons of the KPII intersect, a plethora of patterns can emerge. We focus here on the ones most frequently observed in shallow waters. To do this, fix $\epsilon=0.2, d^{2}=1 / 3$ and $u_{0}=1$ and choose two line solitons with specific parameters, so that the angle of interaction will lead to different patterns. We evolve these initial waves

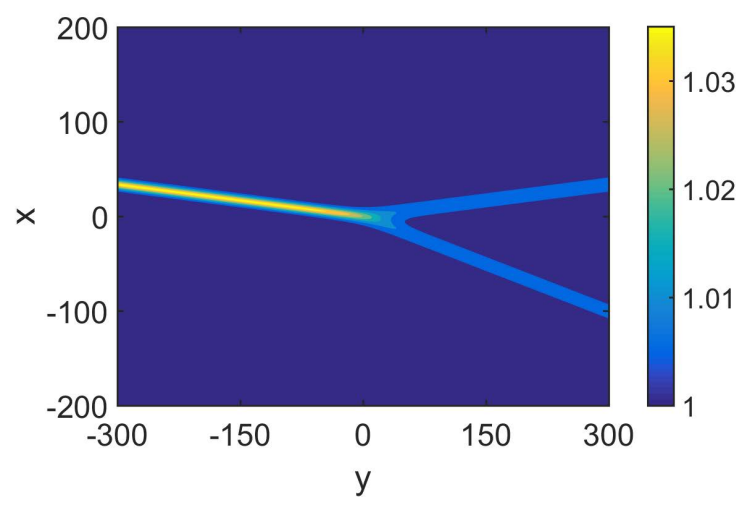

FIG. 1: A Y-type interaction for $2 \kappa_{1}=\kappa_{2}=1$ and $\lambda_{1}=3 \lambda_{2}=\frac{1}{4}$.

up to $t=600$ so that they have enough time to interact. In Fig. 1 we show the resonant interaction of two line solitons resulting into a Y-type wave. The parameters leading to these interactions are summarized in the figure captions.

$\mathrm{X}$-type waves can also emerge, as shown in Fig. 2 These structures are essentially discriminated by their "stems": a short, intermediate and a long stem are respectively depicted in the top, middle and bottom panels of Fig. 22, notice that the long stem's height is higher than that of the incoming line solitons. In addition, we can produce long stem interactions where the stem height is lower than the tallest incoming line soliton, cf. Fig. 3. We refer to these patterns as H-type.

While we chose the above patterns as they appear more frequently in water, other more exotic, web-like structures are also supported by the KPII equation [25-27], and may -in principle- also be produced in optics. Furthermore, these solutions, while approximate, also hold well beyond the smallamplitude limit: similar results (not shown here) were obtained even for $\epsilon=0.7$, with the only additional effect being emission of noticeable radiation. This is due to the robustness of KPII solitons, which is also verified by the observation of these patterns in shallow water, even after the waves break [6].

From the viewpoint of experiments, observations of antidark solitons were reported in Refs. [15, 28]. The Y-, X- and $\mathrm{H}$-waves may be observed experimentally using a setup similar to that of Ref. [15]. In particular, one may employ at first a cw laser beam, which is split into two parts via a beam-splitter. One branch goes through a cavity system to form a pulse (as happens in typical pulsed lasers); this pulse branch undergoes phase-engineering, i.e., passes through a phase mask so that the characteristic phase jump of the antidark soliton is inscribed. Then, the $\mathrm{cw}$ and the phase-engineered pulse are incoherently coupled inside the nonlocal medium, e.g., a nematic liquid crystal, described by Eqs. (1)-(2). This process forms one antidark soliton, as in Ref. [15]. To observe Y-, Xor H-patterns predicted above, two such antidark solitons have to be combined inside the crystal. The angle between the two incident beams, which should be appropriately chosen so that a specific pattern be formed, can be controlled by a rotating mirror in one of the branches. 

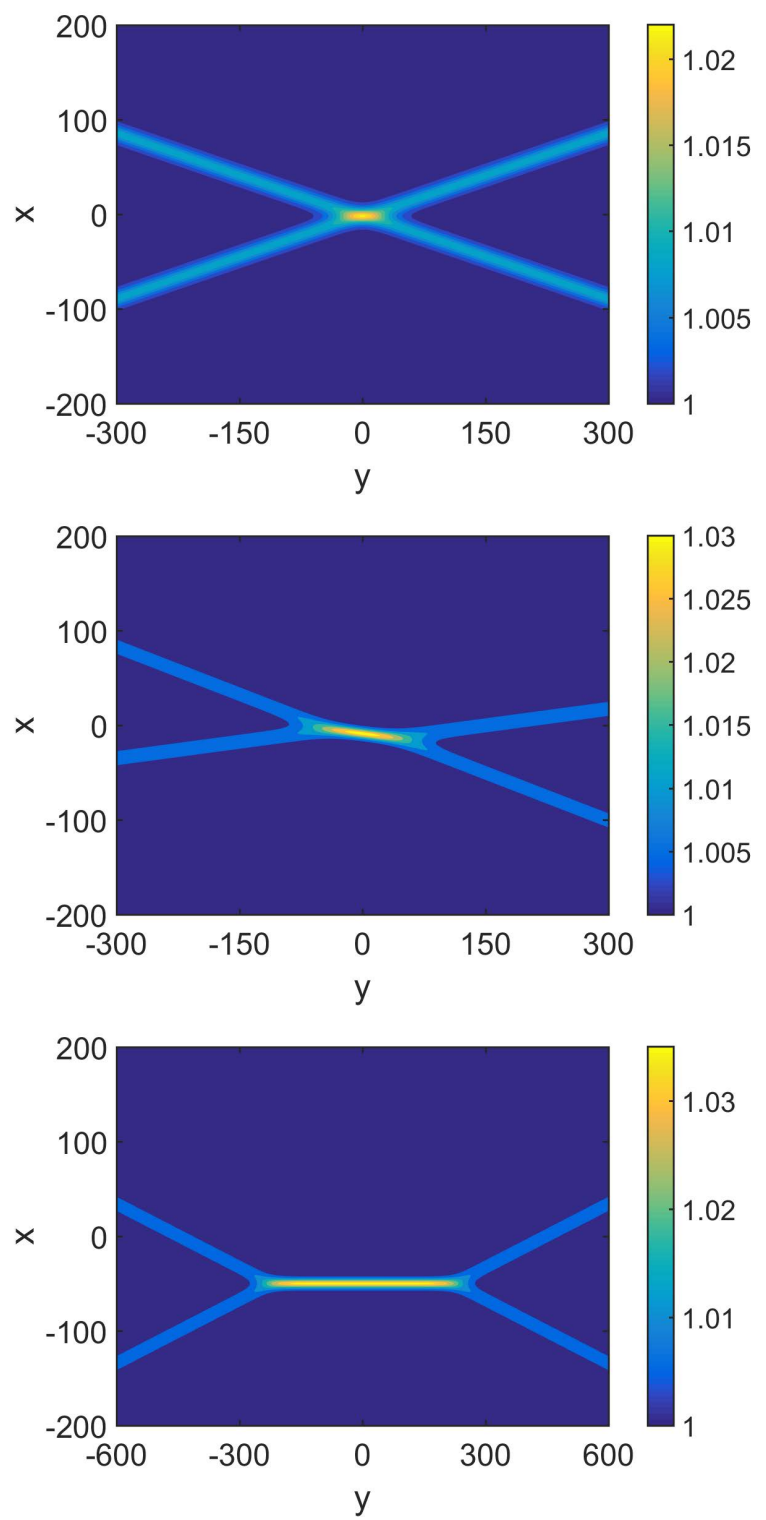

FIG. 2: (Color online) X-type interactions. Top: short stem, for $\kappa_{1}=$ $\kappa_{2}=\frac{1}{2}$ and $\lambda_{1}=-\lambda_{2}=\frac{2}{3}$. Middle: intermediate stem, for $\kappa_{1}=\kappa_{2}=\frac{1}{2}, \lambda_{1}=-\frac{1}{4}-10^{-2}$, and $\lambda_{2}=\frac{3}{4}$. Bottom: long stem, for $\kappa_{1}=\kappa_{2}=\frac{1}{2}$ and $\lambda_{1}=-\lambda_{1}+10^{-10}=\frac{1}{2}$.

Finally, let us consider the KPI case $(\alpha>0)$. KPI also exhibits line soliton solutions, as above, which are however unstable; it is thus most known for its solution that decays algebraically in both spatial coordinates, i.e., the lump:

$$
\begin{gathered}
U(\mathcal{X}, \mathcal{Y}, \mathcal{T})= \\
4 \frac{-\left(\mathcal{X}+a \mathcal{Y}+3\left(a^{2}-b^{2}\right) \mathcal{T}\right)^{2}+b^{2}(\mathcal{Y}+6 a \mathcal{T})^{2}+1 / b^{2}}{\left[\left(\mathcal{X}+a \mathcal{Y}+3\left(a^{2}-b^{2}\right) \mathcal{T}\right)^{2}+b^{2}(\mathcal{Y}+6 a \mathcal{T})^{2}+1 / b^{2}\right]^{2}}
\end{gathered}
$$

where $a, b$ are free real parameters. Lumps have not yet been observed in water. In Fig. 4, we show a direct simulation for the dark lump, and we refer the reader to the recent work [29] for details on multi-lump solutions and their interactions.

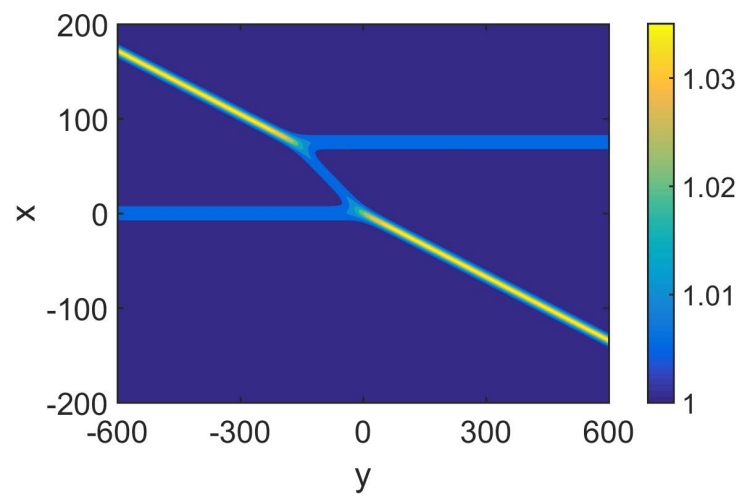

FIG. 3: An H-type interaction with $2 \kappa_{1}=\kappa_{2}=1, \lambda_{1}=\frac{1}{2}-10^{-7}$, and $\lambda_{2}=0$.

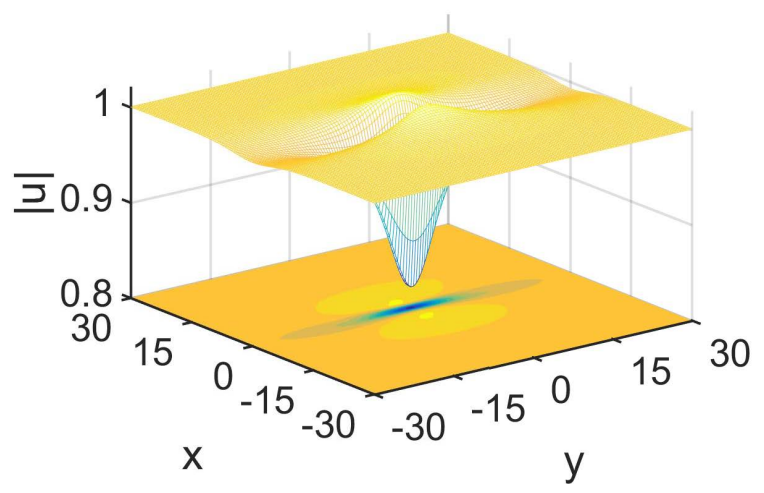

FIG. 4: A typical lump solution with $a=0, b=1$.

Concluding, we have established a homeomorphism between nonlocal nonlinear media and shallow water waves. In particular, we have shown that there exists a surface tension analogue in optics, depending on the nonlocality strength. This was identified from the linear theory and was rigorously analyzed in the fully nonlinear regime, by the asymptotic reduction of a nonlocal NLS system to the KP equations. We demonstrated that, by depending on the effective surface tension, i.e., the degree of nonlocality, novel structures can appear in optical nonlocal media. Thus, fascinating phenomena that appear in water waves can also be observed in optics; an example studied here is the emergence of X-, H-, or Y-shaped waves resulting from the resonant interactions of stable line antidark solitons that were found to exist in strongly nonlocal media. For weakly nonlocal ones, dark lumps were also predicted to occur. The structures predicted in this work may in principle be experimentally observed, either by choosing a material of specific nonlocality or in a specific material altering the magnitude of the cw background, in a setup similar to the one used for he observation of antidark solitons [15].

We thank M. J. Ablowitz, P. J. Ioannou and E. P. Fitrakis for many useful discussions. 
[1] M. J. Ablowitz, Nonlinear dispersive waves: Asymptotic analysis and solitons (Cambridge University Press, Cambridge, 2011).

[2] V. E. Zakharov and E. A. Kuznetsov, Physica D 18, 455 (1986).

[3] T. Dauxois and M. Peyrard, Physics of Solitons (Cambridge University Press, Cambridge, 2006).

[4] M. J. Ablowitz and P. A. Clarkson, Solitons, nonlinear evolution equations and inverse scattering (Cambridge University Press, Cambridge, 1991).

[5] A. Hasegawa and Y. Kodama, Solitons in optical communications (Claredon Press, Oxford, 1995).

[6] M. J. Ablowitz and D. E. Baldwin, Phys. Rev. E 86, 036305 (2012).

[7] C. Rotschild, O. Cohen, O. Manela, M. Segev, and T. Carmon, Phys. Rev. Lett. 95, 213904 (2005).

[8] W. Krolikowski, O. Bang, N. I. Nikolov, D. Neshev, J. Wyller, J. J. Rasmussen, and D. Edmundson, J. Opt. B: Quantum Semiclass. Opt. 6, S288 (2004).

[9] A. G. Litvak, V. A. Mironov, G. M. Fraiman, and A. D. Yunakovskii, Sov. J. Plasma Phys. 1, 60 (1975).

[10] A. I. Yakimenko, Y. A. Zaliznyak, and Y. S. Kivshar, Phys. Rev. E 71, 065603(R) (2005).

[11] C. Conti, M. Peccianti, and G. Assanto, Phys. Rev. Lett. 91, 073901 (2003).

[12] G. Assanto, Nematicons: Spatial Optical Solitons in Nematic Liquid Crystals (New Jersey: Wiley-Blackwell, 2012).

[13] T. Lahaye, C. Menotti, L. Santos, M. Lewenstein, and T. Pfau, Rep. Prog. Phys. 72, 126401 (2009).

[14] D. J. Benney and J. C. Luke, J. Math. and Phys. 43, 309 (1964).
[15] T. H. Coskun, D. N. Christodoulides, Y.-R. Kim, Z. Chen, M. Soljacic, and M. Segev, Phys. Rev. Lett. 84, 2374 (2000).

[16] Y. S. Kivshar and G. P. Agrawal, Optical Solitons: From Fibers to Photonic Crystals (Academic Press, 2003).

[17] N. Ghofraniha, C. Conti, G. Ruocco, and S. Trillo, Phys. Rev. Lett. 99, 043903 (2007).

[18] C. Conti, A. Fratalocchi, M. Peccianti, G. Ruocco, and S. Trillo, Phys. Rev. Lett. 102, 083902 (2009).

[19] V. E. Zakharov and E. A. Kuznetsov, Physics Uspekhi 40, 1087 (1997).

[20] E. A. Kuznetsov, A. M. Rubenchik, and V. E. Zakharov, Phys. Rep. 142, 103 (1986).

[21] E. Infeld, A. Senatorski, and A. A. Skorupski, Phys. Rev. Lett. 72, 1345 (1994).

[22] E. A. Kuznetsov and S. K. Turitsyn, JETP 55, 844 (1982).

[23] D. E. Pelinovsky, Y. A. Stepanyants, and Y. S. Kivshar, Phys. Rev. E 51, 5016 (1995).

[24] Y. S. Kivshar and D. E. Pelinovsky, Phys. Rep. 331, 117 (2000).

[25] S. Chakravarty and Y. Kodama, J. Phys. A: Math. Theor. 41, 275209 (2008).

[26] S. Chakravarty and Y. Kodama, Stud. Appl. Math. 123, 83 (2009).

[27] S. Chakravarty, T. Lewkow, and K.-I. Maruno, Appl. Anal. 89, 529 (2010).

[28] D. Y. Tang, J. Guo, Y. J. Xiang, G. D. Shao, Y. F. Song, L. M. Zhao, and D. Y. Shen, in Photonics and Fiber Technology 2016 (ACOFT, BGPP, NP) (Optical Society of America, 2016), p. JM6A.17.

[29] F. Baronio, S. Wabnitz, and Y. Kodama, Phys. Rev. Lett. 116, 173901 (2016). 